\title{
«SOMOS IGUALES A CUALQUIER OTRA FAMILIA ... ESTAMOS CONVENCIDOS DE QUE SOMOS VERDADEROS PADRES Y DE QUE FORMAMOS UNA AUTÉNTICA FAMILIA». LAS FAMILIAS ADOPTIVAS EN EL CATÁLOGO DE LAS NUEVAS FORMAS FAMILIARES $^{1}$
}

\author{
MARÍA JOSÉ RODRÍGUEZ JAUME \\ Universidad de Alicante \\ MARÍA JosÉ GONZÁLEZ RíO \\ Universidad de Alicante
}

Recibido: 24/03/2014

Aceptado: 12/05/2014

\section{Resumen}

En este artículo abordamos el significado de la familia adoptiva a partir del análisis del discurso de los relatos autobiográficos de madres y padres adoptivos españoles. En un contexto de vacío de cultura adoptiva, las familias adoptivas publican narraciones para ser valoradas como «normales» al tiempo que, en ausencia de modelos de referencia, definen su modelo de familia desdibujando el arquetipo familiar instituido. A partir del método biográfico, aplicamos un doble ejercicio sociológico de (1) deconstrucción ideológica del modelo de familia hegemónico a partir de la (2) construcción del significado que padres y madres adoptivas otorgan a su familia. Las teorías de la familia postmoderna y las teorías feministas postestructuralistas enmarcan el análisis

1. Este artículo se ha realizado en el contexto de los proyectos de investigación $\mathrm{I}+\mathrm{D}+\mathrm{I}$ «El (baby) boom de las adopciones internacionales en España. Una investigación sociológica sobre las familias adoptivas y sus estilos de vida» (CS02009-14763-C03-03) y «Adopciones y acogimientos en España: desafíos, oportunidades y dificultades familiares y sociales durante la infancia y la adolescencia» (CSO2012-39593-C02-01) financiados por el Ministerio de Economía y Competitividad de España. 
crítico del discurso con perspectiva de género con el que es abordado el estudio de estos singulares documentos personales.

Palabras clave: Adopción internacional, familia adoptiva, documentos personales, feminismo posestructuralista, familia postmoderna.

\begin{abstract}
In this article we tackle the meaning of the adoptive family from the analysis of the adoptive Spanish mothers and fathers' speech. In a context empty of adoptive culture, adoptive families publish narrations to be valued as «normal», while as the same time, due to an absence of reference models, they define their family model, blurring the lines of the familiar archetype established. Starting from the biographical method, we apply a double sociological exercise of (1) ideological deconstruction of the hegemonic family model, from the (2) construction of the meaning that adoptive parents bestow upon their family. The postmodern family theories and the poststructuralist feminist ones demarcate the critical analysis of the speech with a gender perspective.
\end{abstract}

Keywords: International adoption, adoptive family, personal documents, poststructuralist feminism, postmodern family. 


\section{Introducción}

Somos iguales a cualquier otra familia (...) estamos convencidos de que somos verdaderos padres y de que formamos una auténtica familia, es un extracto del libro «Padres del deseo, hijos de la esperanza: historia de una adopción en Rusia», la narración autobiográfica de unos padres adoptivos españoles. Maty (empleada de una aseguradora) y Atanasio (médico) deciden, después de once años de novios y tres de casados, que ha llegado el momento de formar una familia. Pero su familia no es una familia como las demás, es una familia adoptiva interracial.

En 2004, fecha en la que Maty y Atanasio adoptaron en Rusia a su hija (Ariadna de tres años) e hijo (Pasha de cuatro años), España se convierte en el segundo país del mundo en adopciones internacionales. Si bien las familias adoptivas en España han adquirido visibilidad, aceptación y relevancia social (Alberdi y Escario, 2003)2 , en el plano de la interacción social, su cotidianeidad aparece condicionada por un modelo de parentesco en donde la consanguineidad determina el ideal de familia hegemónico. Desde este marco cultural dominante, la familia adoptiva es estigmatizada, esto es, es considerada «de segunda» (Miall, 1987) ${ }^{3}$, como la «segunda opción» (March y Miall, 20004; Wegar, 2000) $)^{5}$ o el «último recurso» para ser madres y padres $\left(\right.$ Miall, 1986) ${ }^{6}$. Maty y Atanasio presentan a su familia adoptiva como «auténtica» y se autodefinen como «verdaderos padres» en respuesta a las sanciones informales de las que son objeto pues, tal y como ha destacado la literatura,

2. Alberdi, Inés y Escario, Pilar. Flexibilidad, elección y estilos de vida familiar. Madrid, Ministerio de Trabajo y Asuntos Sociales, 2003.

3. Miall, Charlene. «The stigma of adoptive parent status: perceptions of community attitudes toward adoption and experience of informal social sanctioning». Family Relations, 36 (1) (1987), pp. 34-39.

4. MARCH, Karen y Miall, Charlene. «Adoption as a Family Form». Family Relations, 49 (2000), pp. 359-362.

5. WeGAR, Katarina. «Adoption, family ideology, and social stigma: bias in community attitudes, adoption research, and practice». Family Relations, 49 (2000), pp. 363-370.

6. Miall, Charlene. «The stigma of involuntary childlessness». Social Problems, 33 (1986), pp.268-282. 
la norma social sobre la que se ha construido la «verdadera madre», el «verdadero hijo» y la «verdadera familia» es la que deviene del nexo que establecen los lazos consanguíneos (Alberdi y Escario, 20077; March, 19958; Miall, 19879; Kressier y Bryant, 1996) ${ }^{10}$.

En este artículo abordamos el significado de la familia adoptiva a partir del análisis del discurso de los relatos autobiográficos de madres y padres adoptivos españoles. En un contexto de vacío de cultura adoptiva, las familias adoptivas construyen relatos para que otros puedan valorarlas como «normales» (March, 1995) ${ }^{11}$ al tiempo que, en ausencia de modelos de referencia, definen su modelo de familia desdibujando el ideal de la familia contemporánea. Proponemos, pues, un doble ejercicio: de deconstrucción ideológica del modelo de familia hegemónico, a partir de la construcción del significado y alcance que padres y madres adoptivas otorgan a su forma familiar. Deconstrucción y construcción, dos procesos entrelazados que nos permiten comprender, desde el punto de vista sociológico, la diversidad familiar en las sociedades contemporáneas.

\section{La familia adoptiva desde las teorías feministas postestructuralistas y las teorías de la familia postmoderna}

Para realizar el doble ejercicio de construcción y deconstrucción que proponemos nos apoyamos en las aportaciones de las teorías de la familia postmoderna y las teorías feministas postestructuralistas. Ambos enfoques teóricos comparten el interés por la deconstrucción conceptual de la noción de familia hegemónica y por la construcción terminológica de nuevas formas familiares a partir de un giro metodológico en su estudio. A su vez, los dos enfoques confluyen en el paradigma de la postmodernidad que, en ciencias sociales, se viene aplicando en el estudio de la complejidad y diversidad que asumen los procesos e instituciones sociales, políticas y económicas en las sociedades occidentales avanzadas. El paradigma postmoderno asume la existencia de sistemas no lineales en la sociedad postmoderna. Estos devienen como consecuencia del proceso de individualización que viven las sociedades

7. AlBERDI, Inés y EsCARIO, Pilar. Los hombres jóvenes y la paternidad. Bilbao, BBVA, 2007.

8. MARCH, Karen. «Perception of adoption as social stigma: motivation for search and reunion». Journal of Married and Family, 57(3) (1995), pp. 653-660.

9. Miall, Charlene. "The stigma of adoptive parent status...» Op. cit., p. 3.

10. KRESSIERER, Dana K. y BRYANT, Clifton D. «Adoption as deviance: Socially constructed parent-child kinship as a stigmatized and legally burdened relationship». Deviant Behavior, 17(4) (1996), pp. 391-415.

11. MARCH, Karen. «Perception of adoption as social stigma...» Op. cit., p. 654. 
contemporáneas y con el que adquieren protagonismo las opiniones y decisiones individuales frente a la norma social (Beck y Beck-Gernsheim, 2003) ${ }^{12}$. En la familia, la individualización se traduce en la emergencia de modelos de convivencia postmatrimoniales (Delgado, 1993) ${ }^{13}$ y en modelos de filiación postconsanguíneos (Rodríguez, 2012) ${ }^{14}$ que rompen la hegemonía social e ideológica que la teoría de la modernidad, y sobre todo el funcionalismo, etiquetó como la familia nuclear conyugal. La familia postmoderna «es la etapa en la que la creencia en una evolución lógica de estadios familiares ordenados se ha roto» (Stacey, 1990: 18) ${ }^{15}$. Flaquer añade que, junto a la individualización, un segundo signo distinto de los nuevos tipos familiares en la segunda transición de la familia es el feminismo (1999: 71) ${ }^{16}$.

Las teorías de la familia postmoderna y las teorías feministas postestructuralistas comparten el interés por la deconstrucción conceptual de la noción de familia y por un giro metodológico en el estudio del significado y alcance de la familia. Las aportaciones de Andersen $(1991)^{17}$, Bernardes $\left(1985^{18}, 1993^{19}\right.$, 1999 ${ }^{20}$, Cheal $(1993)^{21}$, Gubrium y Lynott $(1985)^{22}$ y Thorne (1992) ${ }^{23}$ abundan sobre la necesidad de desvelar la «ideología de la familia» (y las implícitas ideologías de la feminidad, masculinidad, maternidad y género), que subyace en el modelo de la familia nuclear, como requisito previo para el desarrollo

12. BECK, Ulrich y BECK-GERNSHEIM, Elisabeth. La individualización. Barcelona, Paidós, 2003.

13. Delgado, Margarita. "Cambios recientes en el proceso de formación de la familia». REIS, 64 (1993), pp. 125-153.

14. RodríGUEZ, $\mathrm{M}^{a}$ José. «Las 'transiciones demográficas' en la segunda modernidad», en Rosa Geldstein y Marta Schufer (eds.): Problemas actuales de salud reproductiva, familia, género y sexualidad, Buenos Aires, PNUD/UNFPA y Biblos, 2012, pp. 83-106.

15. STACEY, Judith. Brave new families. Nueva York, Basic Books, 1990 (citado por D. CHEAL. «Unity and difference in postmodern families». Journal of Family Issues, vol. 14(1) (1993), p. 10.

16. FLAQUER, Lluís. La estrella menguante del padre. Barcelona, Ariel, 1999.

17. ANDERSEN, Margaret. «Feminism and the american damily ideal». Journal of Comparative Family Studies, vol. 22 (2) (1991), pp. 235-246.

18. BERNARDES, Jon. "Family ideology": identification and exploration». Sociological Review, vol. 32 (2) (1985), pp. 257-297.

19. BERNARDES, Jon. «Responsibilities in studying postmodern families». Journal of Family Issues, vol. 14 (1) (1993), pp. 35-49.

20. BERNARDES, Jon. "We must not define "the family" . Marriage and Family Review, vol. 28 (3-4) (1999), pp. 21-41.

21. CHEAL, David. «Unity and difference in postmodern families». Journal of Family Issues, vol. 14 (1) (1993), pp. 5-19.

22. GUBRIUM, Jaber y LYNOTT, Robert. «Family rhetoric as social order». Journal of Family Issues, vol. 6 (1) (1985), pp. 129-152.

23. THORNE, Barrie. Rethinking the family: some feminist questions. Boston, Northeastern University Press, 1992. 
de un enfoque teórico alternativo capaz de analizar la diversidad de la vida familiar contemporánea. En este sentido, ambos enfoques desarrollan una «epistemología de oposición» como estrategia para cuestionar las verdades unitarias y esencialistas.

Por su parte, las aportaciones de Butler $(2001)^{24}$, Levin (1993) ${ }^{25}$, Scanzoni y Marsiglio (1993) ${ }^{26}$ y Scott $(1992)^{27}$ sostienen que para aprehender la diversidad, también de la institución familiar, es necesario desarrollar una «epistemología de y para el cambio» a través un giro metodológico centrado, ahora, en un enfoque constructivista y fenomenológico. La teoría feminista postestructuralista centra su interés en el análisis del lenguaje y del discurso pues son estos los que mejor reflejan el significado social, las relaciones de poder y las múltiples identidades familiares. Los libros autobiográficos que escriben madres y padres adoptivos se convierten en espacios de producción cultural en donde, especialmente las madres, muestran su agencia a través de la experiencia vivida. A través de sus relatos convierten lo «personal» en «político» promoviendo procesos de cambio social. Este posicionamiento fenomenológico es compartido por las reivindicaciones de la teoría de la familia postmoderna. En ella se denuncia la asunción de definiciones en torno a la familia al margen de la experiencia de sus protagonistas planteando que el retorno al sujeto, a su subjetividad, posibilita alcanzar a comprender la diversidad familiar. Las nociones de familia no son estáticas, no solo existen. Su conceptualización se inserta en procesos en donde sus protagonistas las reinterpretan en función de sus acontecimientos vitales (Parry, 2005) ${ }^{28}$.

\section{Los documentos personales de madres y padres adoptivos desde el análisis crítico del discurso y la perspectiva de género}

La deconstrucción ideológica del modelo de familia hegemónica y la construcción subjetiva de la familia adoptiva se aborda, en este trabajo, como

24. BUTLER, Judith. «La cuestión de la transformación social», en Elisabeth Beck-Gernsheim, Judith Butler y Lidia Puigvert: Mujeres y transformaciones sociales, Barcelona, El Roure, 2001, pp. 7-30.

25. LEVIN, Irene. «Families as mapped realities». Journal of Family Issues, vol. 14(1) (1993), pp. 82-91.

26. SCANZONI, John y MARSIGLIO, William. «New action theory and contemporary families». Journal of Family Issues, vol. 14 (1) (1993), pp. 105-132.

27. SCOTT, Joan. «Igualdad versus diferencia: los usos de la teoría postestructuralista». Debate Feminista, vol. 5 (1) (1992), pp. 85-104 (traducción, v.o., 1988).

28. PARRY, Diana. «Women's experiences with infertility: the fluidity of conceptualizations of «family»». Qualitative Sociology, vol. 28(3) (2005), pp. 275-291. 
dos procesos entrelazados que permiten comprender, desde la sociología, la diversidad de estructuras familiares que tienen cabida en las sociedades contemporáneas. Para esclarecer tales procesos, se optó por emplear el enfoque biográfico a partir de los relatos autobiográficos de padres y madres adoptantes. Precisamente, el enfoque biográfico se torna fundamental para el estudio de procesos de cambio que afectan a la estructura biográfica de la trayectoria vital de las personas. De igual modo, resulta útil para analizar experiencias sociales que por su intimidad y complejidad -como las vividas por las y los protagonistas de este trabajo- son difíciles de indagar utilizando otras técnicas de investigación.

Denzin presenta el método biográfico como «el uso sistemático y colección de documentos vitales, los cuales describen momentos y puntos de inflexión en la vida de los individuos. Estos documentos incluyen autobiografías, biografías, diarios, cartas, historias y relatos de vida, crónicas de experiencias personales» (1989: 7) ${ }^{29}$. Aunque el método biográfico cuenta con una larga tradición en el ámbito de la sociología, actualmente hay un gran interés -fuera de la investigación social- por las historias que recogen experiencias vitales de la geste corriente. En palabras de Marinas (2004) ${ }^{30}$, estamos ante el sintoma biográfico que pretende hallar, a través de las palabras, su razón.

En este artículo se toman los documentos personales en los que madres y padres narran en primera persona -sin intervención del investigador- su experiencia con la adopción de sus hijos e hijas en el extranjero. En dos décadas, entre 1990 y 2011 y coincidiendo en el tiempo con la irrupción de la adopción internacional en España, veintiocho historias íntimas y personales de padres adoptivos han visto la luz. El Anexo recoge un cuadro en el que se presentan los veintiocho documentos personales de madres y padres adoptivos a partir del estilo con el que son escritos (diarios, cartas, narración,...), su autoría (madre, padre o relato cruzado) y características de sus estructuras familiares (familias estables, familias reconstituidas, familias monoparentales, familias con filiación mixta y familias con filiación exclusivamente adoptiva).

La autoría de las narraciones autobiográficas sigue el patrón de género observado en la investigación sociológica cualitativa con familias adoptivas

29. Denzin, Norman. Interpretative Biography. Newbury Park, CA, Sage, 1989.

30. MARINAS, José Miguel. La razón biográfica. Ética y política de la identidad. Madrid, Biblioteca Nueva, 2004. 
(March, 199531 y 199732; Miall, 198633 y 1987) ${ }^{34}$ : de las veintiocho historias, diecisiete han sido firmadas por mujeres, seis por hombres, tres adoptan la forma de relato cruzado de la pareja adoptiva y dos han sido escritas por periodistas (una de estas cuenta con la participación de la pareja adoptiva). A pesar de que los relatos aparecen firmados en su mayoría por mujeres, la voz de los padres adoptivos tiene una presencia destacada, lo que sin duda ejemplifica el cambio que la masculinidad hegemónica experimenta en las últimas décadas.

La investigación biográfica se realiza a partir del relato que los protagonistas realizan de su experiencia. El significado que los actores atribuyen a los hechos narrados se convierte aquí en el objetivo central de la investigación. Para su estudio se han aplicado de forma complementaria el análisis crítico del discurso (ACD) y el análisis desde la perspectiva de género. El ACD, siguiendo a van Dijk, aporta una perspectiva crítica sobre la realización del saber. Se centra, en el papel del discurso en la producción y en la reproducción del poder o de la dominación. Toma en consideración las experiencias y la opinión de los miembros de grupos que se encuentran en situación de desigualdad. Desde su planteamiento, «un adecuado análisis del discurso requiere simultáneamente un detallado análisis cognitivo y social, y viceversa, sólo la integración de estas explicaciones puede lograr una adecuación descriptiva, explicativa y, sobre todo, crítica en el estudio de los problemas sociales» (van Dijk 2003:144) ${ }^{35}$. Paralelamente, los documentos personales han sido analizados desde la perspectiva de género, entendido el género como una categoría política forjada por el feminismo, como «ideología transformadora de la realidad» $(\text { Cobo, 2005: 257 })^{36}$. Este análisis implica optar por un enfoque capaz de luchar contra las desigualdades de género y de otra índole, ya que las inequidades condicionan el acceso y el control a recursos y beneficios,

31. MARCH, Karen. «Perception of adoption as social stigma...». Op. cit.

32. MARCH, Karen. «The dilema of adoption reunión: establishing open communication between adoptees and their birth mothers», Family Relations, vol. 46(2) (1997), pp. 99-2015.

33. Miall, Charlene. «The stigma of involuntary...» Op. cit.

34. MiALl, Charlene. «The stigma of adoptive parent status...» Op. cit.

35. VAN DijK, Teun A. «La multidisciplinaridad del análisis crítico del discurso: un alegato en favor de la diversidad», en Ruth Wodak y Michael Meyer: Métodos de análisis crítico del discurso, Barcelona, Gedisa, 2003, pp. 143-177.

36. Сово, Rosa. «El género en las ciencias sociales». Cuadernos de Trabajo Social, 18 (2005), pp. 249-258. 
así como a las opciones vitales, expectativas sociales o de la propia existencia (Botía-Morillas, 2013) ${ }^{37}$.

El análisis de la reconstrucción de los textos se ha llevado a cabo mediante el uso del análisis temático que deviene de la generación inductiva de núcleos temáticos que aparecen de forma repetida y que son destacados por las y los narradores. Se detectan patrones, regularidades en las narrativas sobre lo que, a juicio de quienes escriben, es importante contar o subrayar. Los resultados del análisis se presentan siguiendo el procedimiento de la ejemplificación a través de fragmentos escogidos de los relatos biográficos ${ }^{38}$.

\section{Familias adoptivas en la deconstrucción de la ideología de la maternidad: instinto vs destino}

En sus narrativas autobiográficas, madres y padres adoptivos van introduciendo temas que cuestionan las dimensiones con las que se ha presentado la naturalización de la institución de la maternidad. De forma simultánea desvelan que la ideología de la maternidad es, sobre todo, una construcción social. (Alberdi, 200339; Withers y Thorne, 2013) ${ }^{40}$. De hecho, uno de los temas más recurrentes es que la procreación no es un proceso estrictamente biológico, limitado a la concepción, embarazo y parto.

(...) yo siempre he sido de la opinión de que ser madre no es parir, es criar a un hijo, una tarea que dura toda una vida hecha de muchos momentos y de ir construyendo poco a poco la relación, no únicamente un hecho biológico, y Craig estaba de acuerdo, pero queríamos agotar todas las posibilidades de tener un hijo biológico. (9-Beatriz, 2004: 122)

Es el proceso fisiológico de gestación y parto, lo que ocurre dentro del cuerpo de la mujer, lo que define y diferencia socialmente la maternidad de

37. Botía-Morillas, Carmen. «Cómo diseñar una investigación para el análisis de las relaciones de género. Aportaciones metodológicas», Papers, 98/3 (2013), pp. 443-470.

38. Los verbatims se han trascrito literalmente respetando el estilo y la forma de expresión original. Entre paréntesis se indica la autoría y año de publicación del documento personal. Cuando el documento personal es escrito a dos voces (relato cruzado) se indica entre corchetes quien de la pareja lo ha escrito. La descripción sociodemográfica y familiar de quienes suscriben el verbatim se puede seguir con el número que precede a la referencia de cada uno de ellos que, a su vez, se identifica con su respectivo documento personal en el cuadro que recoge el Anexo.

39. AlbERDI, Inés. «El feminismo y la familia. Influencia del movimiento feminista en la transformación de la familia en España». Arbor, CLXXIV 685 (2003), pp. 35-51.

40. Withers, Marie y THORNE, Barrie. «Las familias y la sociedad en la construcción social del género», en Capitolina Díaz y Sandra Dema (eds.): Sociología y género, Madrid, Tecnos, 2013, pp. 127-152. 
la paternidad (Alberdi y Escario, 200741. Imaz, 2010) ${ }^{42}$ y, en consecuencia, lo que otorga significación e identidad social, específicamente, a la mujer. Cristina relata los sentimientos, emociones y miedos del proceso que le lleva de mujer a madre tomando como referente, nuevamente, el marco de lo biológico. En su narración se subraya que el hecho de no alojar corporalmente a quien será su hijo no implica que este no sea gestado por quien será su madre. Los «hijos del corazón» es una expresión muy recurrente entre los padres adoptivos. La metáfora sirve para expresar que la gestación en la maternidad adoptiva reemplaza al cuerpo, al vientre, por el amor cuyo lugar simbólico corresponde al corazón.

La espera para tener un hijo adoptado, es muy distinta a la espera de las madres biológicas. Ellas van sintiendo como crece su hijo en su vientre: es algo tangible. Pero una madre adoptiva no siente en su cuerpo nada distinto, no tiene nada concreto a lo que agarrarse. (...) Me decía a mí misma: 'Voy a ser madre' y me lo tenía que repetir una y otra vez para convencerme de que era verdad, de que, aunque yo no sintiera nada distinto en mi cuerpo, (...) mi hijo estaba un poquito más cerca de mi. Así es como aprendí algo que dicen los siempre los padres adoptivos: un hijo biológico va creciendo en el vientre de su madre, un hijo adoptado va creciendo en el corazón. (14-Cristina, 2006: 58)

Los discursos nos remiten al amplio campo semántico del que participa el término reproducción. Así, mientras que la filiación biológica se habría construido, esencialmente, a partir de la experiencia con el proceso de concepción, gestación y parto (procreación biológica); la filiación adoptiva pondría el acento en el trabajo de cuidado (crianza). Emerge la moderna «paternidad social» (Beck-Gernsheim, 2003: 16) ${ }^{43}$ en la que los comportamientos reproductivos se desvinculan de los modelos «normales», «biológicos»y «naturales» y en el que lo afectivo reemplaza a la sangre.

El relato de la experiencia vivida con la adopción, especialmente entre quienes buscaron inicialmente la descendencia biológica, evidencia el carácter determinista que para las mujeres encierra la naturalización de la maternidad: «Siempre piensas que tener un hijo es algo que ocurre de manera natural (...), dejas que la naturaleza siga su curso y un día estás embarazada» (9-Beatriz, 2004: 16). La cualidad de «natural» y el carácter determinista que asume la maternidad biológica son ideas que les recuerda, constantemente, el entorno social. Beatriz describe las estrategias de control social que despliega

41. AlBERdi, Inés y EsCARIO, Pilar. Los hombres jóvenes... Op. cit., p.61.

42. IMAZ, Elixabete. Convertirse en madre. Madrid, Cátedra Feminismos, 2010, p. 231.

43. BeCK-GERnSHEIm, Elisabeth. La reinvención de la familia. Barcelona, Paidós, 2003. 
la sociedad sobre la maternidad como una «presión silenciosa construida a base de miradas, de palabras de ánimos de consuelo no dichas por no sacar el tema» (9-Beatriz, 2004: 17). La presión que el entorno y la familia ejercen sobre la maternidad lleva a Beatriz a vivir la imposibilidad de no tener hijos biológicos como un «fracaso».

(...) y cada vez que te ven te preguntan «iY no piensas tener niños?, que tu padre está deseando ser abuelo, desde luego, os lo tomáis con una calma hoy en día...». (...) y no puedes evitar que has fallado a tu marido, a tu familia, a ti misma, que tienes un «defecto de fabricación» y que eres un poco menos completa como persona por no ser capaz de concebir. (9-Beatriz, 2004: 18)

La consideración de que la maternidad deviene de un hecho biológico lleva a cuestionar a aquellas opciones familiares que desafían el carácter natural de la ideología de la maternidad. Las sanciones de las que son objeto las mujeres que llegan a la adopción evidencia el sesgo biológico que regula socialmente el ideal de familia. Sara explica que durante el tiempo de espera de su hija recibió comentarios de su círculo más cercano que venían a demostrar la falta de comprensión: «no entendían por qué me embarcaba en un proceso tan largo y complicado, siendo aún joven, pudiendo aún tener esperanzas de concebir mis propios hijos, según decían» (11-Sara, 2005: 56-57). El cuestionamiento a «salirse de las filas de la normalidad» se problematiza cuando este procede de los servicios que participan administrativamente en el proceso de adopción. El relato de Carmen, que llega a la adopción después de haber tenido tres hijos biológicos, es muy evocador:
A pesar de tener delante un informe muy positivo, cuestionaron nuestra motivación, ya que no acaban de entender porque unos Padres jóvenes, aún fértiles, sin problemas para engendrar de nuevo un hijo biológico, y con tres hijos ya, y teniendo de ambos sexos, querían meterse en esa «aventura». Parece ser que intuían una motivación altruista en nuestro proyecto. (...), cómo es posible pensar que puedan tener una motivación altruista solo por- que elijan la adopción en lugar de volver a tener otro hijo biológico? (18-Car- men, 2007: 29-30)

A través de las narraciones de madres y padres adoptivos también se cuestiona la ideología del instinto maternal según el cual no solo es la biología (la diferencia sexual entre mujeres y hombres) la que determina que las mujeres sean madres sino que son ellas, gestantes, las únicas que pueden trazar un vínculo especial con sus hijos e hijas. El instinto, sustentado a través de los lazos biológicos, determina el convertirse en madre y justifica el rol expresivo de las mujeres. Millán, mientras esperaba el encuentro con su primera hija adoptada explica como «De repente, casi por instinto, me desperté de mi 
letargo. (...) Aquella criatura que acababa de entrar en hotel llorando, de pelo rasurado, cabeza grande, delgada y ojos tristes, era nuestra hija» (17-Angels y [Millán], 2007: 54). Discursivamente, uno de los términos más rememorados por los padres y madres adoptivas es el «destino» con el que reemplazan al «instinto».

Al final del proceso, sin conocerla todavía, quería y deseaba a mi hija con mucha más fuerza que al inicio, (...) simplemente porque deseaba ser madre de una niña que nacería muy lejos de nuestra casa, pero con la que Dios o el destino, o los dos a la vez, me habían destinado a reunirme. (11-Sara, 2005: 20)

Howell y Marre (2006) ${ }^{44}$ han anotado que entre padres y madres adoptivos se desarrollan narraciones en las que incluyen un pensamiento mágico en torno a un discurso basado en similitudes en un escenario de suerte o destino. El sentimiento de predestinación, destino, la coincidencia de un nombre o una fecha con significación para las y los padres adoptantes les hace sentir que su hijo o hija adoptada era para ellos. En este sentido, el destino adquiere la misma función social determinista que el instinto asume en una maternidad y paternidad biológica y evidencia la construcción social que se hace de la maternidad (social y biológica). Obviar el papel que desempeña el «destino» implicaría diferenciar su nacimiento del nacimiento biológico.

\section{Familias adoptivas en la deconstrucción del modelo de masculinidad hegemónico: nuevas paternidades}

La emergencia de la familia moderna se ha explicado a partir de su especialización en labores de reproducción social. Esto es, el paradigma de la modernización se articuló en torno a la dicotomía público-privado que en el orden familiar conllevó la especialización funcional de los roles según sexo (Parsons y Bales, 1955) 45 : los hombres (padres) se situarían en la esfera pública desarrollando el rol instrumental; mientras que el dominio de las mujeres (madres) alcanzaba a la esfera privada en virtud del rol expresivo que les regalaba la naturaleza. A partir de esta ideología de género implícita en el modelo de familia parsoniano, la maternidad es planteada como un hecho natural ligado a la reproducción biológica; mientras que la paternidad aparece vinculada con

44. Howell, Signe y MARRE, Diana. «To kin a transnationally adopted child in Norway and Spain: the achievement of resemblances and belonging». Ethnos, vol. 71(3) (2006), pp. 293-316

45. PARSONS, Talcott y BALES, Robert. Family, socialization and interaction process. Nueva York, Free Press, 1955. 
las responsabilidades que sienten los padres hacia su vida futura y la de su descendencia. En consecuencia, la paternidad es planteada de cara a la sociedad y a la reproducción social (Alberdi y Escario, 2007) ${ }^{46}$. En este contexto, y de forma paralela a la desarticulación de la ideología de la maternidad, los discursos de madres y padres adoptivos también cuestionan el paradigma dominante de masculinidad hegemónico sobre el que se ha sustentado la familia patriarcal. La «sangre», la transmisión patrimonial a través del vínculo consanguíneo, es una de las representaciones sociales instalada en el imaginario colectivo que más se identifica con la identidad masculina patriarcal ${ }^{47}$. Los relatos autobiográficos cuestionan el «nominalismo familiar». Cristina, madre adoptiva monoparental, muestra su desconcierto ante la exigencia de una norma que le obliga a asignar un nombre «ficticio» a un padre que no existe.

[en el consulado español para la inscripción de la hija adoptada en el Registro Civil] (...) el único inconveniente fue la parte del cuestionario de inscripciones en el que hay que poner el nombre del padre adoptivo. María ya me ha dicho que tenía que poner un nombre cualquiera, ya que era obligado rellenar el cuestionario completo. A mi aquello me pareció demencial. ¿Tenía que inventarme un nombre para ponerlo en la casilla de «nombre de padre», a pesar de que todo el mundo sabía que la adopción la realizaba yo sola? ¿Y como se explicaba que hubiera un "padre» y luego, sin embargo, los apellidos de la niña fueran los míos? Tan raro me había parecido aquello que no hice caso a María y dejé esa parte en blanco. Mi asombro fue cuando la funcionaria de la embajada me devolvió el impreso diciéndome, de forma tajante, que tenía que poner un nombre de padre. «isi no hay ningún padre ijesto es una adopción monoparental ${ }^{»}$ (...) Así que, sin protestar más, rellené la dichosa casilla. (...) Curiosamente, unos meses después de nuestro regreso a España, el español, había cambiado al fin la absurda ley que obligaba a las madres solteras a inscribir a sus hijos inventándose el nombre del padre ${ }^{48}$. (14-Cristina, 2006: 173-174)

46. Alberdi, Inés y ESCARIO, Pilar. Los hombres jóvenes... Op. cit., p. 83.

47. En la tradición popular se mantiene la máxima «todo hombre será hombre hasta que haya escrito un libro, sembrado un árbol y tenido un hijo».

48. La normativa a la que se refiere Clara ha estado en vigor desde 1958 hasta 2006. El Reglamento del Registro Civil de 1958 recogía que, en el caso de hijos de padres desconocidos, «el encargado consignará en la inscripción de nacimiento o en otra marginal, en lugar de los nombres del padre o de la madre, otros de uso corriente a efecto de identificar a la persona». La medida se adoptó para evitar la discriminación y oprobio social de los hijos de madres solteras a los que se les asignaba, con anterioridad, el apellido «Expósito». En 1966 la Dirección General de Registros y Notarios explicó el cambio argumentando fines humanitarios pues era una medida que «vela con una apariencia, sin daño para nadie, del que carece de padres desconocidos». 
La legitimidad de los hijos ha sido una de las funciones más importantes del régimen patriarcal. La pérdida de importancia social de la norma de la «ilegitimidad», argumenta Flaquer $(1999)^{49}$, visibiliza la pérdida de poder del patriarca dibujando el emergente modelo de familia postpatriarcal caracterizado por «el papel menguante que desempeña en él el patriarca» (ibíd., p. 17).

Los relatos de las madres adoptivas asumen, en este contexto, no solo los discursos más transgresores respecto la norma social sino que desempeñan un papel central en la transición de sus parejas a la paternidad social. En la totalidad de los documentos personales son ellas las que redefinen, en primera instancia, el sentido de la maternidad a partir de su experiencia con la adopción y son ellas, también, las que participan en la redefinición de la paternidad desvinculándola de lo biológico y natural. Maty, una vez que la pruebas diagnósticas determinaron que eran estériles idiopáticos, contempló la posibilidad de la adopción pero, sin embargo, accedió a someterse a un «largo peregrinaje médico» por complacer a su pareja dado que este, afirma, «no estaba preparado para materializar este alucinante proyecto: adoptar a un niño» (19-[Atanasio] y Maty, 2007: 30). Similar experiencia la relata Beatriz quien después de someterse a varios ciclos de inseminación accede a someterse a un tratamiento de fecundación in vitro pues «Graig no estaba aún convencido del tema de la adopción, quería agotar todas las posibilidades de tener un hijo por métodos más o menos naturales» (9-Beatriz, 2004: 18). La experiencia con la adopción revela la importancia que la «sangre» adquiere en la ideología de la paternidad. El lazo biológico determina, a partir del símbolo de los parecidos, el vínculo que se fraguará entre padre e hijo e hijas pero también les convierte en padres en el plano social. Esta es la transcendencia social de lo biológico en la identidad masculina.

La idea de la adopción ya rondaba entonces en mi mente, conozco casos cercanos de niños adoptados y de familias felicísimas, y siempre he pensado que tener un hijo es mucho más que un hecho biológico, pero a Craig y a mí nos hacía mucha ilusión tener un hijo con sus ojos y mi sonrisa, un niño con talento musical como su padre y el carácter fuerte como mi abuelo, un hijo con sangre escocesa y temperamento español que sacase lo mejor de los dos y que heredase todas las historias, tradiciones y talentos de ambas familias. (9-Beatriz, 2004: 16)

La experiencia con la adopción crea espacios discursivos con los que superar la distancia que un embarazo y parto establece entre lo maternal biológico y

49. FlaQUer, Lluís. Op. cit., p. 33. 
lo social paterno. «Ya estamos embarazados» es una expresión reiterada en los relatos analizados y que sirve para describir un proceso que ahora, desde sus orígenes, será compartido. Mymma, que tuvo su primer hijo después de someterse a dos ciclos de inseminación, cuenta que «Un mes más tarde y llenos de ilusión abríamos el expediente para una adopción internacional. -Nos sentíamos de nuevo embarazados, pero esta vez sería una preñez compartida donde sufríamos los dos los dolores del parto» (15-Mymma, 2006: 10).

La experiencia compartida de formar una familia a partir de la adopción de un menor les lleva a producir discursos en los que los lazos afectivos se desprenden de toda connotación naturalista. De este modo, la sentimentalización que la familia moderna había construido en torno a la ideología de la maternidad y al rol expresivo de la madre, deja de tener su tradicional significación social. Se pasa así de «ser padre» a «tener un hijo»; esto es, se transita desde la acepción social de la paternidad (en cuanto otorgadora de estatus y reconocimiento social) a una concepción de la paternidad personal y con un contenido más emocional y desencadenadora de sentimientos (Alberdi y Escario, 2007) ${ }^{50}$. «Craig está emocionado, tiene los ojos brillantes y me mira embobado, su hijo ya le necesita. -No te puedo explicar lo que siento en estos momentos -me susurra.- No hace falta...- respondo con la voz temblando». (9-Beatriz, 2004: 272)

\section{Familias adoptivas en construcción: iguales pero diferentes}

El término que más evoca la ruptura de la familia adoptiva con el ideal de familia dominante, y que exige a sus protagonistas la redefinición de identidades individuales y sociales, es el de «construcción». El hecho de no participar del sistema hegemónico de filiación consanguíneo, de salirse del trazo unidireccional que ha dibujado la norma social, unido a la ausencia de un modelo de referencia les lleva a definir a su familia en proceso de construcción.

Construir un hogar con un hijo adoptado tiene dificultades añadidas respecto a los hijos biológicos. La primera, vencer los propios miedos y ansiedades, las fantasías sobre las repercusiones que pueda tener su herencia biológica en su vinculación con nosotros y en su futura integración profesional y social. En segundo lugar, aceptar en lo más profundo que somos una familia distinta, pero no diferente de las demás en derechos y obligaciones, en dificultades y recompensas. (21-Alfonso, 2008: 71)

Este proceso de construcción de la familia adoptiva aparece marcado por la experiencia de sentirse socialmente «diferentes». En el relato de Beatriz se

50. AlbERDI, Inés y ESCARIO, Pilar. Los hombres jóvenes... Op. cit., p. 111. 
aprecia la percepción consciente que madres y padres adoptivos tienen sobre las diferencias de su modelo familiar. Los elementos diferenciadores se identifican en la propia ideología dominante según la cual el «verdadero vínculo», el «verdadero amor filial» se establece a partir de la consanguineidad (Miall, $1987)^{51}$.

Si hay algo que uno aprende rápido cuando decide adoptar es el término biológico. (...) existe una cierta tendencia a distinguir entre hijos propios y adoptados, «hijos de verdad»y adoptados, en resumen «hijos-hijos» o premios de consolación. (...) ese matiz del «hijo de verdad» frente a este otro hijo que encuentras, que adoptas, está muy presente en todas las conversaciones. (9-Beatriz, 2004: 57)

A partir de una percepción consciente sobre su realidad diferencial, madres y padres adoptivos despliegan en sus documentos personales una contra-narrativa con la que se enfrentan a la ideología dominante. En el plano discursivo el recurso a la «diferencia» aparece inevitablemente ligado con el de la «igualdad». Atanasio y Maty muestran esta aparente ambivalencia discursiva en un intento por reivindicar el estatus social de su familia. En su relato se aprecia el diálogo que establecen con «los otros». Reprochan a la sociedad que no consideren la adopción como una verdadera maternidad, "Una maternidad que también da a luz, pero sin contracciones y sin ayuda de anestesia» (19-[Atanasio] y Maty, 2007: 87) y recriminan la incomprensión y soledad con la que han transitado por su paternidad adoptiva.

Ahora somos iguales a cualquier otra familia, sin mayores diferencias. Al menos, nosotros lo vemos así; no sabemos cómo nos observarán los demás. Lo que antes nos preocupaba, ahora lo hemos relativizado y nos importa mucho menos. Por eso estamos convencidos de que somos verdaderos padres y que formamos una auténtica familia. (19-[Atanasio] y Maty, 2007: 144)

Tal y como sucede en el discurso de familias gays y lesbianas (Weston, 2003) ${ }^{52}$, los padres adoptivos incluyen en sus narraciones la ideología del amor, en contraposición a la ideología y familia consanguínea, como forma de legitimar otras formas de establecer el parentesco. El amor, en sus relatos, se presenta como un estado de ánimo interior pero, fundamentalmente, como un vínculo social. El amor es la ideología sobre la que los padres adoptivos definen su paternidad y con la que caracterizan a su familia.

51. Miall, Charlene. «The stigma of adoptive parent status...» Op. cit., p. 3.

52. WESTON, Kath. Las familias que elegimos. Lesbianas, gays y parentesco. Barcelona, Bellaterra, 2003. 
Cada vez choca menos ver preciosas niñas de ojos rasgados de la mano de sus padres españoles y hablando un perfecto castellano; (...) familias felices, completas, normales, como cualquier otra, unidas por lazos más fuertes si cabe que la sangre, familias que han hecho realidad su sueño por encima de las trabas administrativas, de las largas esperas y de los problemas. (...) que mira a su padre español con la misma adoración y cariño con que yo recuerdo haber mirado al mío a su edad (9-Beatriz, 2004: 34-35)

Cuando perciben la ausencia del amor, emerge un sentimiento de infelicidad bajo la sospecha de que, probablemente, este solo tiene cabida entre quienes comparten la misma sangre, o bien, que el amor que pueden llegar a sentir puede no ser un amor filial «verdadero». El relato de Atanasio y Maty deja explícito el valor simbólico que socialmente se le atribuye a los lazos consanguíneos en la definición del auténtico amor filial.

Lo que no entendía era la tristeza que veía en su rostro. Suponía que debía de estar feliz, ya que nuestro sueño de ser padres se había cumplido. ¿Qué pasaba, entonces? (...) Un día vino una amiga a la casa y se dio cuenta de lo poco animada que estaba Maty. -¿Qué te ocurre?- le preguntó la amiga. -iTú querías a tu hija cuando nació? -le respondió enseguida. -Ahora que tiene casi un año la quiero, pero al principio era un sentimiento confuso. Maty rompió a llorar. -Joder, y pensaba que no los quería porque eran adoptados. Menudo peso se quitó de encima aquella tarde. Después de tantos cursos y sesiones, nadie se atrevió a decirnos: no los vais a querer; los desearéis, pero no los vais a querer desde el principio. Ahora es cuando se confirma que el roce hace el cariño, por encima de los lazos de sangre (19-[Atanasio] y Maty, 2007: 130 y 132)

La diversidad de la institución familia contemporánea se enmarca en la segunda transición de la familia en donde esta se define, no por su forma, sino por su contenido (Flaquer, 1999) ${ }^{53}$. Las familias adoptivas, a partir de la ideología del amor en la que se sustentan, fundamentan su cotidianeidad en patrones de funcionamiento y no en el de formación. Sus contra-narrativas respecto a los símbolos de la sangre, y la institucionalización del amor como elemento vertebrador de las familias, les lleva a defender una ideología de familia más flexible y atenta a la diversidad familiar.

[27 de enero de 2003] Cuando mi hijo me pregunte si somos una familia a pesar de que él o ella no haya salido de mí, de que yo sea morena y española, su padre de sangre escocesa y él venga de país frío y hermoso, le explicaré que hay familias altas, bajas, gordas, flacas, familias que hablan alto o que casi no hablan, que se quieren en varios idiomas o que entienden sólo uno, familias de colores, familias pequeñas o con cientos de primos, tíos y hermanos

53. FLAQUER, Lluís. Op. cit., p. 63. 
esparcidos por todo el país, familias que viven todos juntos bajo el mismo techo y otras con dos casas, familias con más de un padre o una madre, con hermanos parecidos pero distintos, familias que se llevan bien y otras que discuten. -Porque lo que de verdad hace a una familia (...) es el cariño, el respeto, la ternura, los lazos que unen a los que la forman y que van más allá de la piel o la sangre, (...) que se van trenzando en el tiempo hasta hacer una cadena que ata sin apretar los corazones. (9-Beatriz, 2004: 78-79)

Por último, los documentos personales de madres y padres adoptivos crean espacios de producción cultural que dibujan no solo estrategias de resistencia ante las constricciones que impone la norma (estructura) sino que también perfilan actos de transformación social. Las narraciones autobiográficas muestran el papel activo de, especialmente, las madres adoptivas erigiéndose como agentes activos de cambio. La agencia que asumen las madres adoptivas no se ciñe al ámbito estrictamente familiar. En sus discursos se apunta al deseo de contribuir a la transformación de la sociedad.

Al principio, cuando estaba esperando, me sentía como si continuamente tuviera que estar justificando mi decisión delante de las personas. Afortunadamente con la llegada de Sara eso cambió. (...) Pienso que es mi mejor aliada en esta cruzada en que mi vida se ha convertido, en la lucha por cambiar un poco el mundo, por ampliar la visión de la gente, por hacerles ver cómo el amor es poderoso y cómo puede formar familias de distintas maneras, en distintos lugares, por caminos mágicos y misteriosos. (...) La sonrisa de mi hija dice tantas cosas que ya no siento que tenga que justificarme ante nadie. Ella es lo primero, ella y la bonita historia que estamos escribiendo juntas y que tal vez contribuya a hacer este mundo pequeño un poco más grande y tolerante. Eso es lo importante y lo demás es muy secundario, poco importa lo que digan lo que hagan aquéllos que no entienden. (11-Sara, 2005: 57)

\section{Conclusiones}

Los documentos personales escritos por madres y padres adoptivos no solo dan cuenta de la experiencia vivida con la adopción de sus hijos e hijas sino que, también se convierten en espacios en donde reivindicar el estatus social de su familia adoptiva frente a la norma social que les estigmatiza. La vigencia en occidente de un sistema de parentesco definido a partir de la ideología de la familia biológica conlleva a que las familias no constituidas a partir de vínculos consanguíneos hayan sido socialmente construidas como «anormales». En este contexto, madres y padres adoptivos enuncian contra-narrativas con las que reconciliarse con el guión biográfico, identitario y social que impone el modelo hegemónico de familia biológica.

El desarrollo de estas narrativas alternativas aparece marcado por un doble proceso entrelazado. Por un lado, articulan discursos que sirven para 
deconstruir la ideología de la familia hegemónica, así como las ideologías de maternidad, paternidad y género implícitas en la misma. A partir del cuestionamiento de la norma social señalan algunos de los elementos en torno a los cuales la institución familiar se ha construido socialmente. Así, defienden que la procreación no es un fenómeno estrictamente biológico limitado al embarazo y al parto; denuncian la naturalización implícita en el ejercicio de la maternidad; subrayan la problematización que se hace de las formas familiares ajenas al determinismo e instinto biológico; reivindican el rol expresivo y el espacio privado para los padres cuestionado el modelo hegemónico de masculinidad; y dan cuenta de la pérdida de poder del patriarca en la institución familiar contemporánea.

Paralelamente, en sus discursos y a partir de la experiencia de sentirse socialmente «diferentes», describen el proceso de construcción de sus familias adoptivas. Los elementos diferenciadores se identifican en la propia ideología dominante según la cual el «verdadero amor filial», la «verdadera familia», se establece a partir de la consanguineidad. Madres y padres adoptivos incluyen en sus narraciones la ideología del amor, en contraposición a la ideología y familia consanguínea, como forma de legitimar otros modos de establecer el parentesco, como un vínculo social, como elemento que define su maternidad y paternidad y como signo distintivo de su familia. Sus contra-narrativas respecto a los símbolos de la sangre y la institucionalización del amor como elemento vertebrador de las familias, les llevan a defender una ideología de familia más flexible, basada en el contenido y no en la forma familiar, y atenta a la diversidad familiar. 
Anexo I. Documentos personales de madres y padres adoptivos (1990-2011)

\begin{tabular}{|c|c|c|c|c|c|}
\hline \multirow{2}{*}{$\mathrm{n}^{\mathrm{o}}$} & \multirow{2}{*}{ Documento Personal } & \multirow{2}{*}{ Tipo } & \multirow{2}{*}{ Autoría } & \multicolumn{2}{|c|}{ Familia } \\
\hline & & & & A & $\mathrm{F}$ \\
\hline 1 & $\begin{array}{l}\text { Cuando la familia crece. Historia de una adopción } \\
\text { (1990) }\end{array}$ & EP & $\mathrm{H}$ (Per) & $\mathrm{M}$ & AM \\
\hline 2 & Una adopción en la india (1997) & $\mathrm{N}$ & $\mathrm{H}$ & M & AM \\
\hline 3 & Carta a mi hijo adoptado (2001) & $\mathrm{C}$ & $\mathrm{M}$ & $\begin{array}{l}\mathrm{DM} \\
\mathrm{PH}\end{array}$ & Mix \\
\hline 4 & Diari d'una mare en tràmit (2002) & $\mathrm{D}$ & M & $\begin{array}{l}\mathrm{DM} \\
\mathrm{PH}\end{array}$ & A \\
\hline 5 & Ja venim, Jian (2002) & NC & $\mathrm{P}$ & $\begin{array}{c}\mathrm{DH} \\
\mathrm{M}\end{array}$ & A \\
\hline 6 & Els nens vénen de Sibèria (2003) & $\mathrm{N}$ & $\mathrm{P}$ & $\begin{array}{c}\mathrm{DH} \\
\mathrm{M}\end{array}$ & Mix \\
\hline 7 & $\begin{array}{l}\text { Tú, nuestro sueño. Crónica de } ~ \\
\text { internacional (2003) }\end{array}$ & LD & $\mathrm{M}$ & & \\
\hline 8 & En busca de Irena (2003) & $\mathrm{N}, \mathrm{F}$ & $\mathrm{H}$ & $\begin{array}{c}\mathrm{DH} \\
\mathrm{M}\end{array}$ & Mix \\
\hline 9 & Al final del camino. Diario de una adopción (2004) & D, @ & $\mathrm{M}$ & $\mathrm{DH}$ & A \\
\hline 10 & $\begin{array}{l}\text { Mi pequeña rubí: (Xiao Jing). Ave } \\
\text { adopción (2004) }\end{array}$ & $\mathrm{N}$ & $\mathrm{H}$ & $\mathrm{M}$ & A \\
\hline 11 & Venida de la lluvia (2005) & $\mathrm{N}, \mathrm{D}$ & M & $\mathrm{M}$ & $\mathrm{A}$ \\
\hline 12 & Històries d'una adopció int & & $\mathrm{M}$ & $\mathrm{M}$ & $\mathrm{A}$ \\
\hline 13 & $\begin{array}{l}\text { Mariposas. Realidad y sentimientos de una madre } \\
\text { adoptiva (2006) }\end{array}$ & $\begin{array}{c}\mathrm{N}, \mathrm{D} \\
\mathrm{CD}\end{array}$ & $\mathrm{M}$ & M & A \\
\hline 14 & En busca de Clara. Diario de una adopción (2006) & $\mathrm{D}$ & $\mathrm{M}$ & Mon & $\mathrm{A}$ \\
\hline 15 & Diario de una adopción (2006) & $\mathrm{D}, \mathrm{N}$ & $\mathrm{M}$ & $\mathrm{M}$ & Mix \\
\hline 16 & Un mar d'amor: diari d'una doble adopció (2006) & $\mathrm{D}$ & $\mathrm{M}$ & & \\
\hline 17 & $\begin{array}{l}\text { Una luz vino de Oriente. Historia de una doble } \\
\text { adopción (2007) }\end{array}$ & $\begin{array}{c}\text { EP, } \\
\text { NC, F }\end{array}$ & $\begin{array}{l}\text { M (Per) } \\
\text { P }\end{array}$ & $\begin{array}{c}\mathrm{DM} \\
\mathrm{M}\end{array}$ & Mix \\
\hline 18 & Mei Xiu Duo. Historia de una adopción (2007) & $\mathrm{D}, \mathrm{F}$ & $\mathrm{M}$ & $\begin{array}{c}\mathrm{DM} \\
\mathrm{DH} \\
\mathrm{M}\end{array}$ & Mix \\
\hline 19 & $\begin{array}{l}\text { Padres del deseo, hijos de la espera: historias de } \\
\text { una adopción en Rusia (2007) }\end{array}$ & $\mathrm{N}$ & $\mathrm{P}$ & M & AM \\
\hline 20 & $\begin{array}{l}\text { Padres adoptivos. Cómo afrontar el proceso y la } \\
\text { educación de nuestros hijos (2007) }\end{array}$ & $\mathrm{N}$ & M & M & A \\
\hline 21 & La adopción: un viaje de ida y vuelta (2008) & $\mathrm{NC}, \mathrm{I}$ & $\mathrm{H}$ & $\mathrm{M}$ & $\mathrm{AM}$ \\
\hline 22 & Dos billetes de ida, cuatro de vuelta (2008) & $\mathrm{N}$ & $\begin{array}{l}\mathrm{H} \\
\mathrm{P}\end{array}$ & M & AM \\
\hline 23 & $\begin{array}{l}\text { Alumbrando un sueño: la atribulada historia de una } \\
\text { adopción (2009) }\end{array}$ & $\mathrm{N}$ & $\mathrm{H}$ & $\mathrm{M}$ & AM \\
\hline 24 & $\begin{array}{l}\text { Un sueño hecho realidad: la historia de tu adopción } \\
\text { (2009) }\end{array}$ & $\begin{array}{c}\mathrm{N}, \mathrm{D} \\
\mathrm{F}\end{array}$ & M & M & A \\
\hline 25 & $\begin{array}{l}\text { Indómito y entrañable. El hijo que vino de fuera } \\
(2010)\end{array}$ & $\mathrm{N}$ & M & $\mathrm{M}$ & A \\
\hline
\end{tabular}




\begin{tabular}{|c|l|c|c|c|c|}
\hline \multirow{2}{*}{$\mathbf{n}^{\mathbf{0}}$} & \multicolumn{1}{|c|}{ Documento Personal } & \multirow{2}{*}{ Tipo } & \multirow{2}{*}{ Autoría } & \multicolumn{2}{|c|}{ Familia } \\
\cline { 5 - 7 } 26 & Cuando yo no estaba, mamá me contó que (2010) & $\begin{array}{c}\text { N, D, } \\
\text { F }\end{array}$ & M & M & Mix \\
\hline 27 & El equipaje de Elena (2010) & N, D & M & M & Mix \\
\hline 28 & Ser madre, saberse madre, sentirse madre (2011) & N & M & Mon & A \\
\hline
\end{tabular}

Fuente: elaboración propia.

Nota (abreviaturas): Documento personal: se indica título y año de publicación. El número que precede al título de algunos documentos personales se asocia con el orden de aparición de los verbatims así como a las referencias de estos libros que se recogen en el texto; Tipo (clasificación del documento personal según estilo narrativo): EP=Entrevista Periodística; $\mathrm{N}=$ Narración; NC=Narración Cruzada; $\mathrm{C}=\mathrm{Carta} ; \mathrm{D}=$ Diario; LD=Libro Digital; F=Fotos; I=Ilustraciones hijas; @=Incluye texto de emails; $C D=I n c l u y e ~ C D$ de cuentos; Autoría: M=Mujer; H=Hombre; P=Pareja; Per=Periodista; Familia: A=Alianza: M=Matrimonio; DM=Divorcio Mujer; DH=Divorcio Hombre; PH=Pareja de Hecho; Mon=Monoparental. En el momento de la publicación ocho de estos libros reflejan la estructura de una familia reconstituida. En estos casos, la relación que mantienen los progenitores de la historia familiar adoptiva aparece subrayada ( $\mathrm{M}$ o $\mathrm{PH})$. El estado civil previo y que motivó la creación de una familia reconstituida $(\mathrm{DH}$ y/o DM) se indica previamente; F = Filiación: A=Adopción; AM=Adopción Múltiple; Mix=Biológicos y Adoptivos.

\section{Referencias bibliográficas}

ANDERSEN, Margaret. «Feminism and the american damily ideal». Journal of Comparative Family Studies, vol. 22(2) (1991), pp. 235-246.

Alberdi, Inés. «El feminismo y la familia. Influencia del movimiento feminista en la transformación de la familia en España». Arbor, CLXXIV 685 (2003), pp. 35-51.

- y ESCARIO, Pilar. Flexibilidad, elección y estilos de vida familiar. Madrid, Ministerio de Trabajo y Asuntos Sociales, 2003.

- Los hombres jóvenes y la paternidad. Bilbao, BBVA, 2007.

BECK, Ulrich y BECK-GERNSHEIM, Elisabeth. La individualización. Barcelona, Paidós, 2003.

BECK-GERNSHEIM, Elisabeth. La reinvención de la familia. Barcelona, Paidós, 2003.

BERNARDES, Jon. " "Family ideology": identification and exploration». Sociological Review, vol. 32(2) (1985), pp. 257-297.

— «Responsibilities in studying postmodern families». Journal of Family Issues, vol. 14(1) (1993), pp. 35-49.

— «We must not define "the family" . Marriage and Family Review, vol. 28(3-4) (1999), pp. 21-41.

BOTíA-MoriLlas, Carmen. «Cómo diseñar una investigación para el análisis de las relaciones de género. Aportaciones metodológicas», Papers, nº 98/3 (2013), pp. 443-470. 
BUTLER, Judith. «La cuestión de la transformación social», en Elisabeth BeckGernsheim, Judith Butler y Lidia Puigvert: Mujeres y transformaciones sociales, Barcelona, El Roure, 2001, pp. 7-30.

CHEAL, David. «Unity and difference in postmodern families». Journal of Family Issues, vol. 14(1) (1993), pp. 5-19.

Сово, Rosa. «El género en las ciencias sociales». Cuadernos de Trabajo Social, 18 (2005), pp. 249-258.

Delgado, Margarita. «Cambios recientes en el proceso de formación de la familia». REIS, no 64 (1993), pp. 125-153.

DENZIN, Norman. Interpretative Biography. Newbury Park, CA, Sage, 1989.

FLAQUER, Lluís. La estrella menguante del padre. Barcelona, Ariel, 1999.

GUBRIUM, Jaber y LYNOTT, Robert. «Family rhetoric as social order». Journal of Family Issues, vol. 6(1) (1985), pp. 129-152.

Howell, Signe y MARRE, Diana. «To kin a transnationally adopted child in Norway and Spain: the achievement of resemblances and belonging». Ethnos, vol. 71(3) (2006), pp. 293-316.

IMAZ, Elixabete. Convertirse en madre. Madrid, Cátedra, Feminismos, 2010.

KRESSIERER, Dana K. y BRYANT, Clifton D. «Adoption as deviance: Socially constructed parent-child kinship as a stigmatized and legally burdened relationship». Deviant Behavior, vol. 17(4) (1996), pp. 391-415.

LEVIN, Irene. «Families as mapped realities». Journal of Family Issues, vol. 14(1) (1993), pp. 82-91.

MARCH, Karen. «Perception of adoption as social stigma: motivation for search and reunion». Journal of Married and Family, vol. 57(3) (1995), pp. 653-660.

- «The dilema of adoption reunión: establishing open communication between adoptees and their birth mothers», Family Relations, vol. 46(2) (1997), pp. 99-2015.

- y Miall, Charlene. «Adoption as a Family Form». Family Relations, no 49 (2000), pp. 359-362.

MARINAS, José-Miguel. La razón biográfica. Ética y política de la identidad. Madrid, Biblioteca Nueva, 2004.

Miall, Charlene. "The stigma of involuntary childlessness». Social Problems, n³3 (1986), pp. 268-282.

- «The stigma of adoptive parent status: perceptions of community attitudes toward adoption and experience of informal social sanctioning». Family Relations, vol. 36(1) (1987), 34-39.

PARSONS, Talcott y BALES, Robert. Family, socialization and interaction process. Nueva York, Free Press, 1955.

PARRY, Diana C. «Women's experiences with infertility: the fluidity of conceptualizations of 'family'». Qualitative Sociology, vol. 28(3) (2005), pp. 275-291. 
RODRÍGUEZ, Ma José. «Las "transiciones demográficas" en la segunda modernidad», en Rosa Geldstein y Marta Schufer (eds.): Problemas actuales de salud reproductiva, familia, género y sexualidad, Buenos Aires, PNUD/UNFPA y Biblos, 2012, pp. 83-106.

SCANZONI, John y MARSIGLIO, William. «New action theory and contemporary families». Journal of Family Issues, vol. 14 (1) (1993), pp. 105-132.

ScotT, Joan. «Igualdad versus diferencia: los usos de la teoría postestructuralista». Debate Feminista, vol. 5(1) (1992), pp. 85-104 (traducción, v.o., 1988).

THORnE, Barrie. Rethinking the family: some feminist questions. Boston, Northeastern University Press, 1992.

VAN DiJK, Teun A. «La multidisciplinaridad del análisis crítico del discurso: un alegato en favor de la diversidad», en Ruth Wodak y Michael Meyer: Métodos de análisis crítico del discurso, Barcelona, Gedisa, 2003, pp. 143-177.

WEGAR, Katarina. «Adoption, family ideology, and social stigma: bias in community attitudes, adoption research, and practice». Family Relations, $n^{\circ} 49$ (2000), pp. 363-370.

WESTON, Kath. Las familias que elegimos. Lesbianas, gays y parentesco. Barcelona, Bellaterra, 2003.

Withers, Marie y THORNE, Barrie. «Las familias y la sociedad en la construcción social del género», en Capitolina Díaz y Sandra Dema (eds.): Sociología y género, Madrid, Tecnos, 2013, pp. 127-152. 